\title{
La vocazione territoriale come mito razionalizzante
}

\author{
Piero Mastroberardino** Giuseppe Calabrese ${ }^{* * *}$ \\ FLORA CORTESE
}

\begin{abstract}
Obiettivo del paper: Il lavoro, al fine di contribuire al dibattito sui processi di governance del territorio, ha l'obiettivo di proporne una visione 'situazionista' alternativa alla visione prevalente, qualificata come 'unitaria' o 'sistemica'.

Metodologia: Il lavoro, di taglio teorico-concettuale, pone a confronto due paradigmi, entrambi pienamente riconducibili alle discipline manageriali, che forniscono una differente chiave di lettura interpretativa di cosa sia il territorio e, conseguentemente, di quali siano $i$ processi attraverso i quali si delinea la governance di un territorio.

Risultati: Lo spunto è la rivisitazione del concetto di 'vocazione territoriale' che, da elemento pseudo-oggettivo e 'naturalmente' osservabile e condivisibile da tutti gli attori di un certo territorio e in un certo momento storico, diviene "mito razionalizzante", ovvero un costrutto intersoggettivo privo dell'aura di oggettività e di funzionalità agli interessi del territorio e frutto, seppure solo in parte intenzionale, dell'azione strategica di una coalizione pro-tempore dominante.

Originalità e limiti della ricerca: L'opzione situazionista, elemento di originalità del lavoro, invita a prendere le distanze da modelli predittivi ex ante. Il deficit predittivo, tuttavia, pur palesandone un limite operativo, non ne qualifica un limite epistemologico in quanto, parafrasando Hayek, esprime una consapevole e caratterizzante rinuncia alle "pretese di conoscenza" tipiche degli approcci neo-positivisti.

Implicazioni pratiche: La rilettura dei processi di governance di un territorio proposta ridimensiona la portata dei modelli di management strategico e di marketing territoriale e propone la tortuosa strada dell'esplorazione delle strategie - cooperative e conflittuali messe in atto da attori elo coalizioni locali per perseguire propri interessi.
\end{abstract}

* Nel presente lavoro, frutto della comune elaborazione degli Autori, Piero Mastroberardino ha curato in particolare i parr. 1 e 6, Giuseppe Calabrese i parr. 2 e 3, Flora Cortese i parr. 4 e 5.

** $\quad$ Ordinario di Economia e Gestione delle Imprese - Università degli Studi di Foggia e-mail: p.mastroberardino@unifg.it

*** Ricercatore di Economia e Gestione delle Imprese - Università degli Studi di Foggia e-mail: g.calabrese@unifg.it

**** Dottore di Ricerca in Economia e Governo dell'Impresa - Università degli Studi di Foggia e-mail: f.cortese@unifg.it 
Parole chiave: territorio; governance; vocazione territoriale; mito razionalizzante; potere; quadro istituzionale

Purpose of the paper: The paper aims to contribute to the debate on the territory governance processes by giving an alternative vision, called 'situationist', compared to the prevailing one, defined as 'unitary' or 'systemic'.

Methodology: In this theoretical paper we compare two paradigms, both belonging to the management studies, which provide a different view of what is the 'territory' and, consequently, what are the processes of governance of a territory.

Findings: The idea is to revisit the concept of 'territorial vocation' that, from objective, observable and shared by all the actors of a certain territory and in a certain historical moment, becomes a 'rationalizing myth'. An intersubjective construct not assisted by an aura of objectivity and functionality to the interests of the territory. The result, influenced by unintended effects, of the strategic action of a pro-tempore dominant coalition.

Originality and limits of the study: The situationist perspective, point of originality of this work, is far from predictive models. This operational limit, however, does not also qualify an epistemological limit. Paraphrasing Hayek, it expresses a conscious and characteristic renunciation to the 'pretence of knowledge' typical of neo-positivist approaches.

Practical implications: The different insight into the territorial governance processes debunks models of strategic management and place marketing and proposes the winding road of exploration of the strategies - cooperative and conflictual - enacted by actors and / or local coalitions to pursue their interests.

Key words: territory; governance; territorial vocation; rationalizing myth; power; institutional framework

\section{Riflessioni introduttive}

Le profonde trasformazioni che caratterizzano l'economia mondiale stimolano gli studiosi delle scienze sociali, i manager e i pubblici amministratori a sperimentare nuove prospettive di osservazione atte a interpretare le logiche emergenti su cui poggiano, da un lato, i vantaggi competitivi delle imprese, dall'altro, i vantaggi comparati dei territori.

La letteratura manageriale è pressoché unanime nel rappresentare una sorta di sinergica ciclicità, virtuosa in alcuni casi e viziosa in altri, tra sviluppo/declino dell'impresa e sviluppo/declino del territorio, in un processo nel quale imprese e territori co-evolvono essendo, reciprocamente, gli uni risorse per la competitività degli altri (Valdani e Ancarani, 2000). Altresì, c’è ampia convergenza nel riconoscere che le fonti della competitività, tanto delle imprese quanto dei territori, si siano progressivamente traslate da fattori tangibili a fattori intangibili.

In questa prospettiva il territorio è stato efficacemente definito come uno "spazio relazionale, complesso, unico e difficilmente imitabile” (Rullani, 1999, p. 25), superando qualsiasi accezione legata allo spazio fisico e includendo connessioni culturali e sociali. Una simile definizione, a nostro avviso, ponendo l'accento sull'unicità e la difficile imitabilità, invita altresì a riflettere circa l'applicabilità di modelli di governo del territorio che ambiscano a livelli elevati di generalità e 
astrattezza e che, per dirla con Hayek, includano ampie "pretese di conoscenza" (Hayek, 1989).

D'altro canto anche i modelli d'impresa hanno manifestato notevoli cambiamenti verso livelli crescenti di disintegrazione verticale, variamente etichettati in letteratura - impresa 'estesa', impresa 'dispersa', impresa 'diffusa' - e tutti protesi a indicare che attività dapprima svolte da una medesima organizzazione si riallocano in distinte entità (imprese e non) multi-territorializzate ma, ad un tempo, territorialmente radicate.

Il processo di globalizzazione, dunque, se da un lato produce la frantumazione delle 'tessere' generando 'mosaici' di creazione di valore sempre più ampi, dall'altro genera - quasi paradossalmente, e negando l'ipotesi della perfetta fungibilità del territorio - un rafforzamento dei processi di concentrazione territoriale e nuove forme di radicamento locale (Trigilia, 2005), mostrando la debolezza strategica del modello di sviluppo dell'impresa basato sulla mera delocalizzazione produttiva.

A fronte di un simile scenario, il tema della governance del territorio è affrontato, a parere di chi scrive, con un approccio che rischia di oscillare tra ingenuità e ipocrisia, finendo per adattarsi comodamente alle numerose e seducenti 'mode' manageriali e consulenziali che sul tema abbondano.

Da una parte, tale tema tende ad essere inquadrato nell'alveo delle cosiddette politiche industriali. Si assiste così alla proliferazione di copiosi e referenziati studi scientifici sulla 'vera' vocazione del territorio, che determinano strategie top-down di sviluppo economico, sociale e culturale, e relative 'cabine di regia' ideate e progettate razionalmente da adhocrazie 'intelligenti' di policy makers illuminati dalla scienza, che danno forma a piani strategici di sviluppo territoriale presentati in affollate conferenze di servizi. Piani dei quali, a distanza di qualche anno - in alcuni casi di qualche mese - non resta che il malinconico website realizzato 'per l'occasione'.

Dall'altra, ricorrendo in maniera estensiva ad analogie con le scienze biologiche, s'illustrano (o forse si auspicano) spontanei processi ordinatori, fenomeni di 'autoorganizzazione', forme di democrazia diretta e di auto-governo locale basato su un sussulto di cittadinanza in grado di attivarsi e dar vita, secondo logiche bottom-up, a processi culturali e politici virtuosi predestinati a ridurre ad unum la molteplicità di interessi e azioni dei soggetti operanti sul territorio, in forza di meccanismi di allineamento delle scelte individuali che, per quanto accuratamente descritti, continuano ad apparire come una sorta di black-box.

Entrambe queste traiettorie rinviano ad un comune paradigma, prevalente negli studi di management, che definiamo 'unitario' (Burrell e Morgan, 1979, p. 204) e che, pur con notevoli differenze tra l'opzione della cabina di regia e quella dell'auto-governo locale, interpreta il territorio come entità collettiva reificata, dotato di propria identità e di un pensiero strategico in grado di generare ordine, armonia e integrazione. In una parola, il territorio è raffigurato come un 'sistema' rispetto al quale assumono valenza locuzioni del tipo mission e vision, vocazione, posizionamento, risorse e competenze, stakeholder, intelligenza, etica, etc. tutte declinate con riferimento al territorio. 
La vocazione territoriale, collocata in una simile cornice sistemica, appare come un elemento auto-evidente, esprimente la sintesi delle risorse, delle competenze e delle capacità potenziali di un territorio, derivanti dal suo passato e vincolanti per il suo futuro, da implementare in una sorta di implicita e sempre valida strategia di differenziazione nella competizione tra territori.

Alla luce delle brevi considerazioni più sopra riportate, il presente lavoro si pone l'obiettivo di proporre una diversa chiave di lettura - definita 'situazionista' - utile alla comprensione dei processi di governance del territorio e alternativa alla visione prevalente. In particolare, il lavoro prende spunto dalla rivisitazione del concetto di vocazione territoriale che, da elemento pseudo-oggettivo e 'naturalmente' osservabile e condivisibile da tutti gli attori in opera in un certo territorio e in un certo momento storico, diviene "mito razionalizzante" (Meyer e Rowan, 2000), ovvero un costrutto intersoggettivo, una creazione, seppure non priva di effetti inintenzionali, frutto dell'azione strategica di una coalizione pro-tempore dominante (Cyert e March, 1963; Thompson, 1967; Mastroberardino, 2006; 2010). Privata dell'aura di oggettività e di funzionalità agli interessi del territorio, la vocazione territoriale è collocata sul piano dei condizionamenti di ordine materiale e simbolico che inducono negli attori di quel territorio credenze, schemi e pratiche di comportamento.

\section{II legame tra impresa e territorio negli studi di management: cenni}

Il legame tra l'impresa e il territorio è da sempre al centro degli studi economici e, più in generale, dell'intero alveo delle scienze sociali, sia nella prospettiva macro - grazie al contributo di geografi, sociologi, studiosi dello sviluppo, economisti generali ed economisti industriali - che micro - con l'opera precipua degli economisti d'impresa.

Non senza qualche elemento di semplificazione, il territorio può essere inteso come una meta-risorsa inclusiva di un'ampia serie di pre-condizioni - materiali e immateriali - favorevoli ovvero ostative allo sviluppo economico e sociale. Lo stesso titolo di questo XXIV Convegno annuale di Sinergie definisce il territorio come 'giacimento' di vitalità per l'impresa, accezione analoga a quella di territorio come 'forziere' di valori già emersa in altri recenti autorevoli contributi (Baccarani e Golinelli, 2010, p. IX). Questa accezione del territorio come asset appare come un lungo fil rouge che connette la teoria del vantaggio competitivo delle nazioni (Porter, 1991) agli studi di Marshall sulle 'economie esterne' (Marshall, 1919), fino a risalire alla teoria del vantaggio comparato, elaborata da Ricardo circa due secoli fa (Ricardo, 1817, pp. 85 e ss.).

Nel nostro Paese, caratterizzato dal fenomeno dei sistemi produttivi locali, si registra su questo tema una lunga e fruttuosa serie di contributi. In primis, sul concetto di distretto industriale (Becattini, 1979; 2000; Becattini e Rullani, 1993; Varaldo, 1979; 2006; Varaldo e Ferrucci, 1997) definito come "una rete complessa e inestricabile di economie e diseconomie esterne, di congiunzioni e connessioni di 
costo, di retaggi storico culturali, che ravvolge sia le relazioni interaziendali sia quelle più squisitamente interpersonali" (Becattini, 1987, p. 47).

Poi, sul concetto di rete come modello inter-organizzativo di sviluppo dell'impresa sul territorio (Lorenzoni, 1990; 1992; Lipparini, 1997; Rullani, 1989, 1993; 1999).

Accanto agli studi di carattere più generale se ne ritrovano altri che, condividendo l'approccio resource-based, approfondiscono traiettorie verticali, nell'ambito del settore turistico e, in particolare, nel filone del destination management (Pechlaner e Weierman, 2000; Franch, 2002; Bellagamba et al., 2007) qualificando i concetti di 'sistema turistico locale' (Mastroberardino, 2004; Sciarelli, 2007), 'sistema territoriale' (Martini, 2005) e 'sistema locale di offerta turistica' (Rispoli e Tamma, 1995). Nella medesima prospettiva, infine, si colloca la letteratura di marketing territoriale (Kotler et al., 1993; Ancarani, 1999; Paoli, 1999; Caroli, 1999; Biggiero e Sammara, 2002) che focalizza l'attenzione sul profilo culturale dei territori come elemento distintivo del vantaggio competitivo, connesso agli effetti 'reputazionali' del brand territoriale (Valdani e Jarach, 1998; Valdani e Ancarani, 2000; AA.VV., 2006; Liguori, 2007).

\section{Vocazione territoriale e sviluppo locale. II punto di vista del paradigma 'unitario'}

Il paradigma 'unitario', coerentemente con quanto propone riguardo alle organizzazioni - e tra queste all'impresa - si caratterizza per l'interpretazione del territorio come sistema, strutturato in sistemi di livello inferiore e collocato, a sua volta, in sistemi di livello superiore, connettendo la totalità dei soggetti in una rappresentazione compiuta - e a primo acchito affascinante - del funzionamento della società (Mastroberardino, 2010).

Dal punto di vista epistemologico tale impostazione, richiamando la locuzione di dominio della teleologia (Wright, 1988), opta per un idealtipo di razionalità sistemica, prediligendo le logiche della spiegazione funzionalista in una lettura collettivista-olista dei fenomeni sociali (Burrell e Morgan, 1979; Mastroberardino, 2006, p. 82). Nella "tensione fondamentale" (Capra, 1997, p. 27) fra le parti e il tutto, la prospettiva 'olistica' e il connesso pensiero 'sistemico' focalizzano l'attenzione sugli aggregati sociali, portatori di fini istituzionali propri (in primis, la sopravvivenza del sistema), sovraordinati rispetto a quelli degli attori che li popolano. Accade così che "ai concetti collettivi corrispondono effettive realtà che istituiscono, plasmano e normano l'individuo (...) la cui esistenza e le cui azioni sarebbero inesplicabili senza tali realtà collettive: stato, nazione, chiesa, esercito, sistema economico, ecc." (Antiseri, 1996, p. 441).

Ecco che il territorio si presenta come un sistema distinto, più o meno compiuto, a seconda dello stadio raggiunto in un'ideale traiettoria evolutiva scandita dalla maturità della funzione di governo. Un'entità collettiva che prevale rispetto agli attori (individuali o collettivi) che ne fanno parte, collocati nel ruolo di componenti 
più o meno dotate di requisiti sistemici. Il territorio 'sistema' diviene soggetto, attore strategico, chiamato a individuare un percorso razionale di valorizzazione delle proprie risorse per perseguire obiettivi di sopravvivenza e di competitività, rispondendo a una serie di leggi, o principi generali, quali sinergia, competizione spaziale, domanda di mobilità, ordine e base di esportazione (Camagni, 1998).

All'ipotesi del territorio come attore strategico se ne aggiunge una seconda che vede gli attori locali orientati al 'bene comune', rispetto al quale le ragioni della collaborazione e della convergenza sono destinate a prevalere - pena la morte del territorio - sugli interessi e sulle finalità individuali, conducendo quel sistema verso un percorso di sviluppo armonioso, vantaggioso per tutti, etico e sostenibile.

Si produce così una rilevante semplificazione dei reali processi decisionali, di creazione del valore e - soprattutto - di distribuzione del valore, sia a livello di governo del territorio che delle organizzazioni in esso operanti, presentando una visione più prossima al 'come dovrebbe essere' o al 'come si vorrebbe che fosse'.

Approfondendo ulteriormente la letteratura manageriale, la convergenza di carattere epistemologico sul paradigma 'unitario' si declina in due prospettive: l'una bottom-up, o dell'auto-governo del territorio; l'altra top-down, o dell'organo di governo del territorio.

La prima prospettiva evidenzia dinamiche di auto-organizzazione e capacità emergenti di governance in grado di generare spontanei processi di auto-governo caratterizzati dall'ampia partecipazione dei portatori d'interesse locali. A questi processi di aggregazione spontanea di prossimità geografica è stato ricondotto il modello tradizionale del distretto industriale territorialmente localizzato, settorialmente specializzato e privo di 'impresa guida'. Questa prospettiva, rinviando al cosiddetto paradigma della complessità (Prigogine e Stengers, 1981; Morin, 1993; Waldrop, 1995; Taylor, 2005; Bocchi e Ceruti, 2007), interpreta il territorio come un sistema complesso, caratterizzato dall'emergere di alcune proprietà non presenti in nessuno dei suoi componenti e, soprattutto, da dinamiche evolutive non lineari note come processi di auto-organizzazione (Bertuglia e Staricco, 2002). Ulteriori elementi caratterizzanti i sistemi complessi autoorganizzantisi sono la causalità reciproca (e non lineare), l'indeterminazione (e non il determinismo), il focus sulle relazioni (e non sugli oggetti), un comportamento emergente bottom-up (e non dettato e pianificato top-down), la non trasferibilità dei modelli evolutivi (la trasferibilità dei modelli evolutivi) (Faggioni e Simone, 2009, p. 6).

Secondo questa impostazione, grazie a processi omeostatici di auto-regolazione, il territorio evolve evitando di convergere verso uno stadio di completo ordine e, allo stesso tempo, non precipitando verso il disordine. L'evoluzione prende forma stabilizzando "una condizione armoniosamente contraddittoria, intermedia tra l'eccesso di organizzazione interna e di sintonia tra i sottosistemi, che porterebbe il tutto di cui essi sono parte alla cristallizzazione e quindi alla rigidità $e$ all'immobilismo, e l'eccesso di conflittualità interna, che farebbe precipitare il sistema nel caos (...) L'orlo del caos può essere allora considerato come quel giusto grado di disordine tra i sottosistemi funzionali che permette al sistema di avere 
buone prestazioni e di modificarsi senza collassare" (Tagliagambe e Usai, 2009, p. 187). Nel nostro Paese questa impostazione ha dato luogo al filone 'territorialista' (Magnaghi, 2005; 2007) che interpreta il territorio come un sistema vivente, la cui identità è costruita intorno ad una specifica 'coscienza di luogo'. Il territorio, dunque, "non è lo spazio geografico né il suolo della pedologia, ma un soggetto vivente ad alta complessità, esito di processi coevolutivi sinergici fra insediamento umano (organizzato su basi culturali) e ambiente (organizzato su basi geologiche e biologiche)" (Magnaghi, 2006, p. 1).

Sull'altro versante del paradigma 'unitario', la prospettiva top-down pone l'attenzione sull'organo di governo del territorio (mono o multi-livello, mono o multi-agente) che esercita la vitale funzione di government (Pernice, 1999) orientando le condotte degli attori in gioco. In questa logica sono stati collocati i cluster tecnologici sviluppatisi nei settori ad alta intensità di ricerca e caratterizzati dalla presenza di una funzione di regia e programmazione centrale svolta da soggetti pubblici ovvero da grandi imprese private (Bottinelli e Pavione, 2010; Velo, 2011). Inforcando queste lenti, il fenomeno dello sviluppo locale è spiegato come esito di un'articolata e razionale attività di 'progettazione', a partire da una vision che appare chiara nella mente di un designer capace di definirne, secondo un modello di razionalità ex-ante, le finalità strategiche e gli obiettivi operativi, i moduli componenti e le relative funzioni, i meccanismi di differenziazione e l'integrazione tra questi; in una parola, l'equilibrio, sia interno che nei confronti delle istanze provenienti dall'ambiente esterno (Bertalanffy, 1968; Beer, 1973; Emery, 1974; Scott, 1981; Gross e Etzioni, 1987; Golinelli, 2000; 2011).

Il passaggio ulteriore trasla al territorio 'sistema' le medesime routine (teoriche $\mathrm{e}$ pratiche) messe a punto per l'impresa 'sistema' con particolare riferimento alle prospettive offerte dalla Resource Based View (Wernefelt, 1984; Barney, 1991; 2001; Grant, 1991) e dalla Stakeholder Theory (Freeman, 1984; 1994; 2004).

Il territorio, dunque, conquista e mantiene un vantaggio competitivo attraverso la valorizzazione delle sue risorse e competenze distintive. La RBV fa emergere un concetto molto solido di vocazione territoriale come sintesi unica delle specificità locali, ovvero delle risorse strategicamente migliori in quanto dotate di valore, scarse, non imitabili e organizzate. Inoltre, il vantaggio è sostenibile se soddisfa le attese dei numerosi e distinti soggetti e/o organizzazioni (stakeholder) che partecipano, direttamente o indirettamente, al processo di creazione del valore, convergendo verso un comune progetto di sviluppo, capace di co-allineare le rispettive forze in base ad una visione radicata e condivisa dell'identità e del futuro del territorio: "E pluribus unum, dalle molte voci una visione unitaria" (Schillaci e Gatti, 2011, p. 24).

È dunque essenziale la "capacità di creare visioni condivise, sviluppare capacità gestionali e organizzative in grado di selezionare gli obiettivi, formulare le strategie giuste e implementare i percorsi ottimali per perseguirle" (Napolitano, 2008, pp. 3132). Il successo competitivo del territorio necessita di "un'impostazione strategica e una chiara visione manageriale che prevedano una stretta interazione, in primo luogo fra gli attori locali e, in secondo, fra questi e l'insieme delle risorse del 
territorio (naturali, economiche, sociali, imprenditoriali e organizzative)" (Sciarelli, 2007, p. 7)

Il territorio, in estrema sintesi, sopravvive ed è competitivo se, grazie all'azione ordinatrice del suo organo di governo, riesce a qualificare un circuito virtuoso di soddisfazione dei sovrasistemi rilevanti, attrazione delle loro risorse, creazione e distribuzione di valore (Golinelli, 2000; 2002).

Un simile approccio è ostile verso ogni manifestazione di potere collaterale e centrifugo, che appare come fonte di 'disordine' locale, di bieco opportunismo, in contrasto con lo sforzo 'buono e giusto' del regolatore centrale. Laddove questa tendenza convergente non si ravvisa, il riscontro di comportamenti 'devianti' è interpretato come il rischio da correre per effetto delle condotte dei singoli attori o di loro aggregati caratterizzati da selezione avversa e azzardo morale (Della Corte, 2000, pp. 138-141). Queste condotte opportuniste, individualiste, egoiste, riducono il valore potenzialmente generabile da una strategia coerente mettendo a rischio quella che sarebbe stata la strada virtuosa per tutti e palesando l'incapacità a lavorare per il 'bene del territorio', minacciandone la sopravvivenza e la competitività.

E brandendo l'utopia del 'bene del territorio' prendono forma e consenso proposte di sviluppo razionali, 'scientifiche', riconducibili in modo diretto o mediato a forme di ingegneria sociale olistica, che promettono la società perfetta, già qualificata da Popper - e dalla storia - come la negazione della società aperta. L'ingegnere sociale erge il suo piano su una scienza che dovrebbe dirci "quali misure dobbiamo prendere se intendiamo, per esempio, evitare le depressioni economiche, o anche provocare tali depressioni; oppure se intendiamo rendere più equilibrata, oppure meno equilibrata, la distribuzione della ricchezza" (Popper, 2003, p. 44). Ed ecco che per il 'bene del territorio' il piano prescrive che il singolo "debba servire gli interessi del tutto", intendendo per tutto qualsivoglia corpo collettivo, dall'impresa al territorio, dal partito allo stato, dalla nazione alla razza (Popper, 2003, p. 133).

\section{La governance locale nella prospettiva situazionista}

Allontanandosi dalla prospettiva 'unitaria', il territorio perde la sua caratteristica di sistema reificato, preminente rispetto agli attori che lo popolano, per riacquistare le meno utopiche sembianze di 'luogo' di interessi differenti, di strategie divergenti, di interazione, di negoziazione. Questa diversa cornice rivaluta la dimensione specifica del territorio e la "dimensione dinamica, contestuale e pratica dell'agire degli attori” (Ogien e Quéré, 2005). Il territorio appare in continua costruzione, mai compiuto, effetto di una rete di azioni interdipendenti, non definibile da un progetto unitario e in un ben delimitato perimetro politico-amministrativo.

Di conseguenza, lo stesso discorso sullo sviluppo locale non può essere incardinato in una logica bottom up, né in una top down. Superando la tentazione della 'spiegazione' ex ante, lo sviluppo locale non è l'effetto di una causa specifica, non nasce da regolamenti e non può essere astrattamente pianificato, ma assume le 
vesti di un processo negoziato tra attori portatori di interessi differenti, che operano strategicamente per ottenere asimmetricamente risorse, nei limiti ad essi posti dalla cornice istituzionale. Non resta che rassegnarsi a una logica di 'comprensione' ex post del fenomeno, centrando lo studio sull'esplorazione delle dinamiche di governance e delle relazioni di potere che si creano tra gli attori del territorio (Mastroberardino e Nigro, 2009; Mastroberardino et al., 2010b).

La matrice epistemologica situazionista offre al riguardo interessanti spunti di riflessione: affondando le sue radici nella fenomenologia sociale (Berger e Luckmann, 1969) e nell'etnometodologia (Garfinkel, 1967), focalizza l'attenzione sui contesti concreti dell'agire umano nella vita quotidiana, sul ruolo degli attori sociali che con la propria partecipazione contribuiscono alla realizzazione della realtà sociale osservata (Sparti, 2002).

A livello teorico, la governance locale viene inquadrata nella sintesi della prospettiva politica (Crozier e Friedberg, 1978; Pfeffer, 1981; Friedberg, 1994; Crespi, 1999) e neo-micro-istituzionalista (Meyer e Rowan, 2000; Zucker, 2000; Powell e DiMaggio, 2000).

La prospettiva politica centra la sua attenzione su attori sociali per definizione 'interessati', ovvero intenzionalmente tesi a soddisfare propri interessi. Mettendo in campo meccanismi di confronto, cooperazione, conflitto, negoziazione essi mirano ad accrescere la propria influenza e il loro potere, e in questo modo contribuiscono, seppur non del tutto intenzionalmente, a creare, preservare e trasformare gli assetti di governance. Il concetto di potere qui evocato riferisce all'archetipo di 'poterescambio': in qualsiasi campo di azione concreto il potere è libertà di azione, è la capacità dell'attore (individuale o collettivo) di strutturare a proprio vantaggio le interazioni, sfruttando i vincoli e le opportunità offerte dal contesto per conquistare, mantenere e ampliare i relativi margini di autonomia e incertezza (Friedberg, 1994; Calabrese, 2006).

La prospettiva neo-micro-istituzionalista si focalizza, viceversa, sulle cornici istituzionali e sui condizionamenti che queste operano sugli attori sociali che in esse agiscono. Le istituzioni e i loro condizionamenti di ordine materiale e simbolico sono il frutto della stessa opera degli attori che tendono a cristallizzare regole e vincoli per creare un 'ordine' locale. Attraverso un processo di isomorfismo, e al fine di ottenere legittimazione esterna, gli attori della governance locale conformano le proprie condotte agli schemi, alle pratiche e alle procedure delle istituzioni.

Tra azione e istituzionalizzazione affiora la visione di un soggetto che, nel suo agire intenzionale, è vincolato istituzionalmente, ma non rinuncia alla sua natura di attore strategico. I due processi, dell'attore e dell'istituzione, producono, senza soluzione di continuità, la costruzione, la rottura e la ricostruzione della realtà sociale. Da qui una governance locale che, pur cristallizzandosi in un assetto istituzionale definito dai rapporti di forza e dal compromesso tra gli attori, e costrittivo nei confronti degli stessi, non dà mai vita ad un quadro definitivo ed immutabile (Mastroberardino, 2006; 2010; Colasanto et al., 2010; Mastroberardino et al., 2010a; Nigro e Trunfio, 2010; Mastroberardino et al., 2010b). 
In questa prospettiva, la governance locale è frutto di un gioco di potere fondato su continua negoziazione. Il processo negoziato, che si presenta come un'interazione in cui più parti cercano di accordarsi su un risultato reciprocamente accettabile in situazioni di divergenza di interessi (Pruitt, 1981), struttura pro-tempore un certo ordine locale, allo stesso tempo prodotto dell'azione umana e vincolo alla stessa (Giddens, 1990). Se l'ordine sociale è un prodotto delle negoziazioni, l'agire negoziale è a sua volta limitato da una precedente interazione. Regole e vincoli, quindi, da una parte creano ordine e minimizzano l'incertezza, dall'altra portano nuovamente conflitti, contraddizioni, ambiguità (Powell e DiMaggio, 2000).

La governance locale, dunque, non può che essere studiata nella sua dinamica, nel suo processo di strutturazione e ristrutturazione continua in cui si manifesta l'agire degli attori locali: non è un sistema predeterminato che viene subìto dagli attori, ma questi ultimi contribuiscono a crearlo, preservarlo e trasformarlo. Gli assetti di governance del territorio sono l'espressione cristallizzata e codificata di un rapporto di forza e di un compromesso tra i suoi attori e diventano strumento di governo e di regolamentazione della dimensione locale; gli attori, allo stesso tempo $\mathrm{e}$ in funzione della percezione che hanno dei vincoli e delle opportunità di quell'assetto, cercano di limitarne la validità o di modificarla (Cortese, 2010).

\section{La vocazione territoriale come "mito razionalizzante"}

L'inquadramento delle dinamiche di governance locale nella prospettiva situazionista consente di interpretare la vocazione territoriale, a cui la letteratura dà un ruolo centrale in tema di sviluppo del territorio, non più come elemento oggettivo, auto-evidente - "la personalità di un territorio, la sua inclinazione naturale, sociale, culturale ed economica" (Golinelli, 2002) - ma in quanto effetto, solo in parte intenzionale, di un potere di influenza e di orientamento di una coalizione pro-tempore dominante, che si compone di attori pubblici e privati che, con interessi contingentemente convergenti, si uniscono per raggiungere un obiettivo. Questa coalizione, nell'intento di dare un'identità al territorio, strumentale al 'posizionamento' dello stesso e al riconoscimento del suo ruolo di attore collettivo in un contesto più ampio, si lascia guidare dal momento politico, esamina le diverse opzioni a sua disposizione e sceglie un sentiero piuttosto che un altro in virtù delle opportunità che in esso intravede (Mastroberardino, 2010). Le opzioni a disposizione della coalizione sono senz'altro influenzate dalle caratteristiche del territorio sistema sociale nel quale agisce, ma il giudizio di "rilevanza" (Golinelli, 2000) di certe componenti di dotazione del territorio ai fini dell'analisi della vocazione stessa è comunque frutto di un processo strategico degli attori. La distinzione tra territori con potenzialità o inclinazione turistica da quelli destinati ad attività di produzione o altro viene superata in forza di un progetto diverso, al quale vengono associate possibilità di sviluppo del territorio stesso.

La vocazione territoriale appare quindi come una 'costruzione' legata all'esercizio di una forma di potere (Cyert e March, 1963; Thompson, 1967; 
Mastroberardino, 2006; 2010; Mastroberardino et al., 2012) che, opportunamente giustificata, assurge a 'dimensione di senso' pro tempore vigente (Nigro, 2006) per gli attori del territorio. Questi ultimi, ai fini dello stesso riconoscimento di attori del territorio, sono portati a conformare alla vocazione il proprio comportamento, per ottenere legittimazione e risorse. Si pensi a come la possibilità di accedere ai finanziamenti pubblici per lo sviluppo di un determinato territorio passi per il far convergere le attività imprenditoriali verso la vocazione territoriale prescelta.

Come qualsiasi altra 'forma' di ordine sociale, o di istituzionalizzazione, la vocazione è uno strumento in grado di indirizzare prima - o espletare un'attività di formazione, che dir si voglia - e controllare poi le dinamiche di un territorio, il comportamento degli altri attori. Foriera di convinzioni e pratiche socialmente approvate assurge a "mito razionalizzante" (Meyer e Rowan, 2000). È attraverso lo stesso processo di isomorfismo che gli attori contribuiscono a rafforzare la vocazione.

I miti razionalizzanti sono definiti come potenti regole istituzionalizzate, in termini di categorie, classificazioni, aspettative di comportamento, norme che connotano i criteri di efficienza adottati dall'organizzazione. Essi però non sono immutabili, bensì in continuo divenire: gli stessi attori, nel loro agire, e in funzione della percezione che hanno dei vincoli e delle opportunità, tendono a ridiscutere e modificare l'idea di vocazione e il grado del suo condizionamento, sempre nel quadro di quel gioco di potere finalizzato all'ottenimento di maggiori/migliori spazi di azione. I margini di intervento sono funzione del grado di "persistenza culturale": quanto più un atto istituzionalizzato si presenta dotato di un alto grado di formalità, tanto più efficace e duratura nel tempo sarà la trasmissione dei suoi valori culturali ai soggetti che ne fanno parte, e meno libertà sarà lasciata ad essi nella rottura e ricostruzione della cornice di regole e norme (Zucker, 2000). Non sono rari i casi di territori turistici in cui sono stati costruiti poli industriali solo per sfruttare 'l'opportunità' del momento.

La vocazione territoriale appare quindi come una temporanea 'modellizzazione della scena': per il territorio vengono create una vision e una mission, valorizzando alcuni aspetti piuttosto che altri; a ciascuno attore viene assegnato un ruolo, con conseguenti aspettative di comportamento, e per la regolazione dei rapporti tra attori vengono utilizzati schemi e procedure; allo scopo di ottenere la riconoscibilità esterna, infine, si arriva a 'sigillare' con dei marchi anche la correttezza e conformità dei comportamenti agli schemi e alle procedure adottate. L'esempio più immediato sono i marchi collettivi o quelli che afferiscono ai prodotti tipici.

In questo quadro non ha senso parlare di territorio, di governance locale e di sviluppo senza esaminare le dinamiche che in esso avvengono, gli effetti che producono le interazioni tra attori, senza considerare l'azione che anima il semplice 'spazio fisico'. È per questo motivo che le implicazioni sopra descritte non restano, e non devono restare, a livello teorico: se la vocazione non ha una vita ex ante, non è auto-evidente od oggettiva come sostiene la parte dominante della letteratura in materia, la capacità di lettura del contesto da parte degli attori chiave, la comprensione del grado di istituzionalizzazione delle variabili sulle quali operare e 
le capacità negoziali sono fondamentali ai fini dello sviluppo di un territorio (Cortese, 2010).

A livello pratico, l'osservatore è portato a cercare una chiave di analisi, pur nella consapevolezza che la realtà socialmente costruita difficilmente si lascia ridurre ad un modello. Nella lettura degli assetti di governance pro-tempore vigenti occorre tener presente almeno tre dimensioni:

- gli attori e le loro interazioni;

- i valori, le regole e le prassi della cornice istituzionale (identità, capitale sociale e relazionale);

- il contesto esterno, che va oltre il 'locale'.

È evidente come le specificità territoriali, la natura profondamente indeterminata dell'azione umana e il carattere politico, e perciò contingente del fenomeno, portino ad un'irriducibile indeterminatezza degli assetti di governance locale.

I vari modelli di governance o strumenti, veicoli di sviluppo - l'Area vasta, i Progetti integrati, i GAL, i Sistemi turistici locali, i Distretti, i Patti territoriali, i Contratti di area, ecc. - assurgono a tipizzazioni concettuali, sottendendo una dimensione simbolica che dipende dai soggetti che ne esperiscono l'essenza e la portata (Nigro e Trunfio, 2010). Con questo non si intende negare l'importanza del processo di pianificazione dello sviluppo locale, ma ridimensionare la centralità e la portata dei vari modelli, ricollocandola nel processo di costruzione sociale della realtà. La pianificazione assume così i caratteri di "uno schema orientativo di massima, che ha pochi, sfumati requisiti di una mappa, cui seguiranno azioni molto più assimilabili all'apertura di un sentiero durante il cammino, che non ad un percorso su binari già tracciati” (Mastroberardino, 2010).

Ai vari attori coinvolti nella governance locale è chiesto di non fermarsi alla chiara e rassicurante rappresentazione della realtà dei modelli, ma di andare 'oltre gli schemi', di inforcare diverse lenti nella lettura di un fenomeno, di non sottovalutare il grado di istituzionalizzazione delle variabili del contesto e di non focalizzare l'attenzione esclusivamente sulle relazioni tra variabili che i modelli impongono.

\section{Considerazioni conclusive e spunti di ricerca empirica}

La riflessione effettuata sui processi di governance conduce a rappresentare il territorio non più come un 'sistema' unitario, ma come un'arena politica, un set di opzioni strategiche, un potenziale di percorsi di sviluppo la cui attivazione non dipende da 'condizioni iniziali', quale sembra essere il concetto di vocazione territoriale maggiormente condiviso dalla letteratura, ma è esito delle concrete strategie coalizionali di attori concreti che pro-tempore sono capaci di indirizzare le dinamiche di un territorio (Mastroberardino, 2010).

La vocazione territoriale diviene un costrutto intersoggettivo, solo in parte intenzionale, frutto dell'agire strategico dei decision-maker locali, che cercano di ritagliarsi asimmetricamente margini di manovra (e di valore) all'interno di una cornice istituzionale fatta di regole, norme, prassi. 
Si colloca, altresì, sul piano dei condizionamenti di ordine materiale e simbolico che inducono negli attori di 'quel' territorio schemi, pratiche e procedure. Attraverso un processo iterativo di 'evocazioni' successive, tende a oggettivizzarsi sino a divenire strumento, seppure non del tutto rispondente alle iniziali intenzioni della coalizione dominante, di orientamento dell'agire individuale.

La stessa 'identità del territorio', che la letteratura considera diretta emanazione della vocazione territoriale, altro non è che un ulteriore costrutto che, assurgendo a dimensione di senso, rende intelligibili le dinamiche di governance locale, la descrizione, la valutazione delle stesse, ma che è co-costitutivo del territorio stesso (Garfinkel, 1967).

Per quanto concerne la ricaduta empirica, la rilettura delle dinamiche di governance locale in chiave situazionista, enfatizzando il continuum tra i processi di azione e di istituzionalizzazione richiede, coerentemente, l'adozione di un approccio metodologico qualitativo che rivaluti le tecniche e gli strumenti propri dello studio storico ed etnografico dei fenomeni sociali e politici. La finalità di tali percorsi di ricerca empirica non potrà essere, dunque, l'individuazione di leggi generali in grado di spiegare le dinamiche di sviluppo o di crisi dei territori, pena il ricadere nelle esoteriche ma sterili piste già battute dai numerosi 'guru' dell'ingegneria sociale olistica.

Per converso, la ricerca dovrà e potrà esplorare a fondo differenti esperienze e casi, successi e fallimenti, in una logica di comprensione ex post delle concrete dinamiche di azione individuale e coalizionale, abbandonando pretenziose ambizioni di minuziosa pianificazione, progettazione e controllo dello sviluppo di un territorio. $\mathrm{Al}$ contempo, la ricerca dovrà e potrà supportare la policy locale puntando alla diffusione della conoscenza circa gli strumenti e le tecniche di negoziazione, nonché le pratiche di lobbying, uscendo definitivamente dalla coltre di ingenuità e di ipocrisia che le connota.

Infine, la ricerca empirica, più che operare aprioristiche distinzioni tra attori buoni e attori cattivi, tra altruisti ed egoisti, dovrà 'addestrarsi' ed 'addestrare' nella preziosa opera di smascheramento dei giudizi di valore che, abilmente celati in ipotesi di ricerca formalmente coerenti, popolano la nostra letteratura, in merito al rapporto tra impresa e territorio, etica imprenditoriale e responsabilità sociale dell'impresa.

\section{Bibliografia}

AA.VV. (2006), "Le marche collettive per il territorio ed i cluster di imprese. Modelli, casi e strategie di sviluppo", Sinergie, Rapporti di ricerca, n. 23.

ANCARANI F. (1999), "Il marketing territoriale: un approccio per la valorizzazione del territorio", Economia e Diritto del Terziario, n. 1, pp. 79-99.

ANTISERI D. (1996), Trattato di metodologia delle scienze sociali, Utet, Torino.

BACCARANI C., GOLINELLI G.M. (2010), "Per una rivisitazione delle relazioni tra impresa e territorio", Sinergie, n. 84, pp. VII-XIII. 
BARLEY S.R., TOLBERT P.S. (1997), "Institutionalization and structuration: studying the links between action and institution", Organization studies, vol. 18, n. 1, pp. 93-117.

BARNEY J.B. (1991), "Firm Resources and Sustained Competitive Advantage", Journal of Management, vol. 17, n. 1, pp. 99-120.

BARNEY J.B. (2001), "Resource-Based Theories of Competitive Advantage: A Ten Year Retrospective on the Resource-Based View", Journal of Management, vol. 27, n. 6, pp. 643-650.

BECATTINI G. (1979), "Dal settore industriale al distretto industriale. Alcune considerazioni sull'unità di indagine dell'economia industriale", Rivista di Politica ed Economia industriale, n. 1, pp. 7-21.

BECATTINI G., (1987), "L'unità d'indagine", in Becattini G. (a cura di), Mercato e forze locali: il distretto industriale, Il Mulino, Bologna.

BECATTINI G. (2000), Il distretto industriale. Un nuovo modo di interpretare il cambiamento economico, Rosenberg \& Sellier, Torino.

BECATTINI G., RULLANI E. (1993), "Sistema locale e mercato globale", Economia $e$ Politica Industriale, n. 80, pp. 28-29.

BEER S. (1973), L'azienda come sistema cibernetico, Isedi, Milano.

BELLAGAMBA A., BRUNETTI F., PENCARELLI T., VIGOLO V. (2007), "La Letteratura italiana e internazionale sul Destination Management", in Sciarelli S. (a cura di), Management dei sistemi turistici. Strategie e strumenti per la governance, Giappichelli, Torino, pp. 31-83.

BERGER P.L., LUCKMANN T. (1969), La realtà come costruzione sociale, Il Mulino, Bologna.

BERTALANFFY L. VON (1968), General System Theory. Foundations, Development, Applications, Braziller, New York.

BERTUGLIA C.S., STARICCO L. (2002), Complessità, auto-organizzazione, città, Franco Angeli, Milano.

BIGGIERO L., SAMMARA A. (a cura di) (2002), Apprendimento, identità e marketing del territorio, Carocci, Roma.

BOCCHI G., CERUTI M. (a cura di) (2007), La sfida della complessità, Mondadori, Milano.

BOTTINELl L., PAVIONE E. (2010), Distretti industriali e cluster tecnologici: strategie emergenti di valorizzazione della ricerca e dell'innovazione, Giuffré, Milano.

BURRELl G., MORGAN G. (1979), Sociological Paradigms and Organizational Analysis, Heinemann, London.

CALABRESE G. (2006), "Il lobbying alla luce dei modelli organizzativi di ispirazione politica", in Mastroberardino P. (a cura di), Lobbying. Agire tra vincoli, ESI, Napoli.

CAMAGNI R. (1998), Principi di economia urbana e territoriale, Carocci, Roma.

CAPRA F. (1997), La rete della vita. Una nuova visione della natura e della scienza, Rizzoli, Milano.

CAROLI M.G. (1999), Marketing territoriale, Franco Angeli, Milano.

COLASANTO M., MASTROBERARDINO P., NIGRO C. (a cura di) (2010), La negoziazione nelle dinamiche di governance locale, Franco Angeli, Milano.

CORTESE F. (2010), "La negoziazione dello sviluppo locale: lettura e comprensione dell'ambito problematico", in Colasanto M., Mastroberardino P., Nigro C. (a cura di), La negoziazione nelle dinamiche di governance locale, Franco Angeli, Milano.

CRESPI F. (1999), Teoria dell'agire sociale, Il Mulino, Bologna.

CROZIER M., FRIEDBERG E. (1978), Attore sociale e sistema, Etas Libri, Milano.

CYERT R.M., MARCH J.G. (1963), A Behavioral Theory of the Firm, Prentice Hall, Englewood Cliffs, NJ. 
DELLA CORTE V. (2000), La gestione dei sistemi locali di offerta turistica, Cedam, Padova.

EMERY F.E. (a cura di) (1974), La teoria dei sistemi. Presupposti, caratteristiche e sviluppi del pensiero sistemico, Franco Angeli, Milano.

FAGGIONI F., SIMONE C. (2009), "Le declinazioni della complessità. Ordine, caos e sistemi complessi”, Sinergie, n. 79, pp. 3-45.

FRANCH M. (2002), Destination Management. Governare il turismo tra locale e globale, Giappichelli, Torino.

FREEMAN R.E. (1984), Strategic Management. A Stakeholder Approach, Pittman, Boston.

FREEMAN R.E. (1994), "The Politics of Stakeholder Theory: Some Future Directions", Business Ethics Quarterly, vol. 4, n. 4, pp. 409-421.

FREEMAN R.E. (2004), “The Stakeholder Approach Revisited”, Zeitschrift für Wirtschaftsund Unternehmensethik, vol. 5, n. 3, pp. 228-254.

FRIEDBERG E. (1994), Il potere e la regola. Dinamiche dell'azione organizzata, Etas, Milano.

GARFINKEL H. (1967), Studies in ethnomethodology, Englewood Cliffs, Prentice Hall.

GIDDENS A. (1990), La costruzione della società, Edizioni di Comunità, Milano.

GOLINELLI C.M. (2002), Il territorio sistema vitale. Verso un modello di analisi, Giappichelli, Torino.

GOLINELLI G.M. (2000), L'approccio sistemico al governo dell'impresa. I L'impresa sistema vitale, Cedam, Padova.

GOLINELLI G.M. (2011), L'approccio sistemico al governo dell'impresa. II Verso la scientificazione dell'azione di governo, Cedam, Padova.

GRANT R.M. (1991), "The Resource-Based Theory of Competitive Advantage", California Management Review, vol. 3, n. 33, pp. 114-134.

GROSS E., ETZIONI A. (1987), Organizzazioni e società, Il Mulino, Bologna.

HAYEK F.A. VON (1989), "The Pretence of Knowledge", The American Economic Review, vol. 79, n. 6, pp. 3-7.

KOTLER P., HAIDER D.H., REIN I. (1993), Marketing Places, Free Press, New York.

LIGUORI M. (2007), Il destination branding nel governo del territorio, Il Calamaio, Roma.

LIPPARINI A. (1997), "Sistemi territoriali e comunità interorganizzative", in Lomi A. (a cura di), L'analisi relazionale delle organizzazioni, Il Mulino, Bologna, pp. 271-307.

LORENZONI G., (1990), L'architettura di sviluppo delle imprese minori. Costellazioni e piccoli gruppi, Il Mulino, Bologna.

LORENZONI G., (a cura di) (1992), Accordi, reti e vantaggio competitivo, Etas, Milano.

MAGNAGHI A. (a cura di) (2005), La rappresentazione identitaria del territorio. Atlanti, codici, figure, paradigmi per il progetto locale, Alinea, Firenze.

MAGNAGHI A. (2006), "Il territorio come soggetto di sviluppo delle società locali", in Atti del convegno Lo sviluppo in questione: le forme umane della trasformazione, Università di Macerata, Falconara Marittima, 8 e 9 novembre 2006, pp. 1-13.

MAGNAGHI A. (a cura di) (2007), Scenari strategici. Visioni identitarie per il progetto di territorio, Alinea, Firenze.

MARSHALL A. (1919), Industry and Trade. A Study of Industrial Technique and Business Organization, MacMillan, London.

MARTINI U. (2005), Management dei sistemi territoriali. Gestione e marketing delle destinazioni turistiche, Giappichelli, Torino.

MASTROBERARDINO P. (a cura di) (2004), Contributi sul tema dei sistemi turistici locali. Riflessioni sull'area garganica, ESI, Napoli.

MASTROBERARDINO P. (a cura di) (2006), Lobbying. Agire tra vincoli, ESI, Napoli. 
MASTROBERARDINO P., NIGRO C. (a cura di) (2009), Le dinamiche di governance d'impresa. Cenni sull'approccio etnometodologico, ESI, Napoli.

MASTROBERARDINO P. (2010), "La governance del sistema impresa tra istituzionalizzazione e azione del soggetto imprenditoriale", Sinergie, n. 81, pp. 135171.

MASTROBERARDINO P., NIGRO C., CALABRESE G. (2010a), “Azione ed istituzionalizzazione", Azienda Pubblica, n. 1, pp. 15-38.

MASTROBERARDINO P., NIGRO C., CORTESE F., CAROLILlO G. (2010b), "La negoziazione nei processi di sviluppo locale”, in Atti XXXIII Convegno AIDEA, Università Bocconi 21-22 ottobre, Milano.

MASTROBERARDINO P., CALABRESE G., CORTESE F. (2012), "Costrutti, miti e strategie nella comunicazione d'impresa”, Sinergie, n. 88, pp. 17-34.

MORIN E. (1993), Introduzione al pensiero complesso, Sperling \& Kupfer, Milano.

MEYER J.W., ROWAN B. (2000), "Le organizzazioni istituzionalizzate. La struttura formale come mito e cerimonia", in Powell W.W., Dimaggio P.J. (a cura di), Il neoistituzionalismo nell'analisi organizzativa, Edizioni di Comunità, Torino, pp. 5987.

NAPOLITANO M.R. (2008), “Competizione territoriale e marketing urbano”, in Napolitano M.R., Riviezzo A., Marketing e gestione strategica dei centri urbani, Franco Angeli, Milano, pp. 21-48.

NIGRO C. (2006), "Il lobbying alla luce dei modelli organizzativi di matrice istituzionalista", in Mastroberardino P. (a cura di), Lobbying. Agire tra vincoli, ESI, Napoli.

NIGRO C., TRUNFIO M.P. (2010), “Confini, coalizioni e governance nei contesti territoriali. Verso la realizzazione dei sistemi turistici locali", Sinergie, Rapporti di Ricerca, n. 31, pp. 47-89.

OGIEN A., QUÉRÉ L. (2005), Le vocabulaire de la sociologie de l'action, Ellipses, Paris.

PAOLI M. (1999), Marketing d'area per l'attrazione di investimenti esogeni, Guerini \& Associati, Milano.

PECHLANER H., WEIERMAN K. (a cura di) (2000), Destination Management. Fondamenti di marketing e gestione delle destinazioni turistiche, Touring University Press, Milano.

PERNICE I. (1999), "Multilivel Constitutionalism and the Treaty of Amsterdam: European Constitution Making Revisited", Common Market Law Review, n. 36, pp. 703-750.

PFEFFER J. (1981), Power in organizations, Ballinger Publishing Company, Cambridge Mass.

POPPER K.R. (2003), La società aperta e i suoi nemici, vol. 1, Armando Editore, Roma.

PORTER M.E. (1991), Il vantaggio competitivo delle nazioni, Mondadori, Milano.

POWELL W.W., DIMAGGIO P.J. (2000) (a cura di), Il neoistituzionalismo nell'analisi organizzativa, Edizioni di Comunità, Torino.

PRIGOGINE I., STENGERS I. (1981), La nuova alleanza, Einaudi, Torino.

PRUITT D.G. (1981), Negotiation behavior, Academic Press, New York.

RICARDO D. (1817), On the Principles of Political Economy and Taxation, trad. it. (1976), Sui principi dell' economia politica e della tassazione, Isedi, Milano.

RISPOLI M., TAMMA M. (1995), Risposte strategiche alla complessità: le forme di offerta dei prodotti alberghieri, Giappichelli, Torino.

RULLANI E. (1989), "Economia delle reti: i linguaggi come mezzi di produzione", Economia e Politica Industriale, vol. 14, n. 56, pp. 147-150.

RULLANI E. (1993), "La conoscenza e le reti: gli orizzonti competitivi del caso italiano e una riflessione metodologica sull'economia d'impresa", Sinergie, n. 31, pp. 147-186. 
RULLANI E. (1999), "L'impresa e il suo territorio: strategie di globalizzazione e radicamento territoriale", Sinergie, n. 49, pp. 25-31.

SCIARELLI S. (a cura di) (2007), Management dei sistemi turistici. Strategie e strumenti per la governance, Giappichelli, Torino.

SCHILLACI C.E., GATTI C. (2011), "E pluribus unum: intenzionalità collettiva e governo dei sistemi territoriali", Sinergie, n. 84, pp. 21-45.

SCOTT W.R. (1981), Le organizzazioni, Il Mulino, Bologna.

SPARTI D. (2002), Epistemologia delle scienze sociali, Il Mulino, Bologna.

TAGLIAGAMBE S., USAI G. (2009), "Soggetti umani e soggetti collettivi nell'impresa e oltre l'impresa", Sinergie, n. 79, pp. 173-191.

TAYLOR M.C. (2005), Il momento della complessità. L'emergere di una cultura a rete, Codice, Torino.

THOMPSON J.D. (1967), Organizations in Action. Social Sciences Bases of Administrative Theory, McGraw Hill, New York.

TRIGILIA C. (2005), Sviluppo locale. Un progetto per l'Italia, Laterza, Bari.

VALDANI E., JARACH D. (1998), "Strategie di marketing per il territorio: come vendere un'area geografica”, in Perrone V. (a cura di), L'occupazione possibile, Etas, Milano, pp. 113-130.

VALDANI E., ANCARANI F. (a cura di) (2000), Strategie di marketing del territorio. Generare valore per le imprese e $i$ territori nell'economia della conoscenza, Egea, Milano.

VARALDO R. (1979), Ristrutturazioni industriali e rapporti tra imprese, Franco Angeli, Milano.

VARALDO R., FERRUCCI L. (a cura di) (1997), Il distretto industriale tra logiche d'impresa e logiche di sistema, Franco Angeli, Milano.

VARALDO R. (2006), "Il nuovo modello competitivo ed aziendale dei distretti industriali", Economia e Politica Industriale, n. 1, pp. 25-43.

VELO D. (2011), "La varietà dei sistemi locali per l'innovazione emergenti in Europa", Sinergie, n. 84, pp. 5-20.

WALDROP M.M. (1995), Complessità. Uomini e idee al confine tra ordine e caos, Instar Libri, Torino.

WERNERFELT B. (1984), “A Resource-Based View of the Firm”, Strategic Management Journal, n. 5, pp. 171-180.

WRIGHT G.H. VON (1988), Spiegazione e comprensione, Il Mulino, Bologna.

ZUCKER L.G. (2000), "Il ruolo dell'istituzionalizzazione ai fini della persistenza culturale", in Powell W.W., Dimaggio P.J. (a cura di), Il neoistituzionalismo nell'analisi organizzativa, Edizioni Comunità, Torino. 
